\title{
XXXIX.
}

\section{Experimentelle Untersuchungen über die Beziehungen zwischen chemischer Constitution und diuretischer Wirkung in der Puringruppe.}

\author{
$\operatorname{lin}$ \\ Peter Bergell und Paul Friedrich Richter.
}

Zwischen der scheinbar so ausgedehnten Synthese neuer Heilmitiel und der geringen Zahl fostgelegrer Cresetge der Bezichungen zwischen aliemiselier Constitution und pharmakologiseher Nirkung besteht ein Missrerhältniss. Wit Sieherheit wissen wir immerhin, dass in einzelnen Klatssen organiseher Verbindungen, ilenen eine specielle, mehr oder minder ausgeprägte phamakologisehe Wirkung zukomml, "henusche Eingrifle an bestimmten Gruppen des Molecüls auch Veründerungen der Wirkung hervorufen, und zwar mit einer gesetzmïssig erscheinenden Zurerlissigkeit. Wir kennen Schlafmittel, Antipyretica, locale Anästhetica, limetica, bei denen die Einführung einer chemischen Gruppe, oder der Frsat: einer vorbandenen chemischen Gruppe durch eine hestimmte andere, in dem einen Falle die pharmakologische Wirkung aufheht, im anderen rerstäkt. Ton einem Gesetz zu spreehen, seheint jedoch insofern nicht statthaft, als die Beobachtungen immer nur in einer Klasse gültig crscheincn und auch ausserdem nach Art der Schmelzpunktsregehöissigkeiten Ausnahmen aufweisen.

Bei der Vielseitigkeit, welche Anzahl der Derivate und die difforenten Wege der Synthese ermöglichen, ergiebt es sich, dass man nur dort hoffen kann, Beziehumgen zwischen dhenjscher Constitution und Wirkung im Organismus ausgjelig zu erforschen, wo die pharmakodynamische Prüfung exact und ror allen Dingen rinlach geselichen kann. „Einer der Hauptgründe, welher einen Einblick in ten Zusammenhang zwischen Constitution und Wirkmg ershwert, ist offenbar darin zu finder, dass man sich diese Beziehungen zu cinfach vorstelte (Ehrlich: Festschrift für Ernst v. Leyden, S. 651)." Kommt zu der Complizirtheit rein chemischer Bezichungen Langwierigkeit, Schwierigkeit oder anch mangelnde Exactheit pharmakologiseher Prüfungen dazt, so worden auch bei den zahreichsten Experimenten Gosetamissigkeiten sich rophergen. die thatsäehlich bestehen. Die hypnotisehe oder emetische Wirkmer

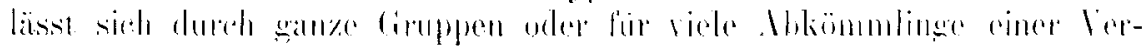


bindung verfolgen; oft rerlangt der liswuls an thierisehen Organismus weniger Zoit und Beobachune: als manche rein ehemische Identiatsreaction.

Im Gegensatz hierzu stand bisher dic pharmakod namische Prüfung der diuretischen Wirkung abemiseher Substanzen. Sie contastirte mit der rorhandenen Aufgabe. Während in der Alkaloidehemie die Sorgfalt pharmakologischer Prüfungen grössere Fortsehritte gemacht hat, als die genauere chemische Kenntniss dieser Köpper, war die Puringruppe analytisch, wie synthetisch aufgeklärt, ohne dass lland in Iland danit eine intimere Kenumiss etwaiger pharmakodynamischer Wirkung ging.

Wir haben nun mit einer neuen einfachen Prüfungsmethode der diuretischen Wirkung boi kranken Vieren ejnen Versnoh gemaeht, zwisclaen einigen genetiseh zusammenhängenden Purinkärpern Vergleiche zu zichen, und ylauben orweisen zu künnen, wie hier die einzelnen synthetischen lingrifle in das Moleciil die pharmakologisehe Wirkmo in eindeutiger Weise beeinflussen. Bei dieser neuen Methode, welehe ron dem einen ron uns (P.F.Richter, Therapie der Gegenwart, Dezember 1904) vor kurzer Zeit mitgetheilt ist, handelt es sich um die Beeinflussung eines im Thierexperimeni hororgerufenen nephritisehen Ilydrops. Spritai man Thieren (Kaninehen) kleine Mengen ron Irannitrat ein, so kann man in kurzer Zeit eine selwere toxische, acute, parenchymatöse Nephritis erzougen. Fïr gowöhnlied, wenigstens bei gecigneter wasterreicher brnälurung der Thiere, ist dieselhe mit leichten Graden ron Ergüsisen in die serösen Höhlen (Pleura und Peritoncum) repbunden. Durch cinc zweckentsprechende Versuchanordnung, dureh die Steigerung der Flïssigkeitszulular mit oder ohne Beigabe ron Salzen, kann man num diese lirgüsse zu ganz auffallend hohen Gralden steigern, so zwal, dass die Oroane der Bauchhöhle röllig rerlagert erseheinen, dass die Pleuren derartig nit serösem Transsudat erfüllt sind, dass die lungen als fast röllig luftlecre Organe ganz in die oberen Theile der Brusthöhle gedrängt sind. Ergüsse in die Bauchböhle von über 100, in die brusthöhle von über 40 cem gebören nicht $\%$ den Seltenheiten -- bei der Kleinheit des Raumes, um den es sich bei 1 his $1 \frac{1}{2}$ Kilo schweren Versuchsthicren handelt, cine ganz colossale Menge, wie jeder zugeben wird. In einer Reihe von Fällen ist auch das Unterhautzelgewebe ödematös; kurz, wir haben ein rälliges Ehenbild des nephritisehen Hydrops beim Mensehen ror uns. Nlles Nähere über Versuchsanordnung siche: I. F. Richter, Therapir der Gegenwart 1904. No. 12; doch sei ausdrïcklich bemerkt, dass nach neuen Versuchen (ef. P. F. Richter, Berl. klin. Wochensebr. 1905) die Beigabe ron Salzen zur Erzeugung ron Hydrops durchans nicht nothwendig ist, sondern die Steigerung der Fiüssigkeitszufuhr allein genügt. In gleicher Weise, wie mit dieser Yethode bishes bewähte Diurelica bereits gepruft sind, haben wir nun cine Roilho neuer Körper untersueht und verglichen. Für dic vorläulige Answahl dienten rein chemische speculationen.

Dir erste Gruppe, die wir untersnchten, waren Homologe des Coffrins, insofern es sich un Terbindungen handelte, bei denen eine der drei 
Methyleruppen durch den ledhylest ersetzt wat. Ls sind dies also drei isomere Körper, und wir kömen die ehemisoben Brychungen sehr äbersichtlich darlegen, indem wir den Purinkem, ohemischen Ísaneen analug, nur mit Ziffern bezciohnen.

Coffein ist 1. 3. 7. Mothyanthin, und nummeriven sich Kohlenstoffund stickstoflathome in folgender lloise:
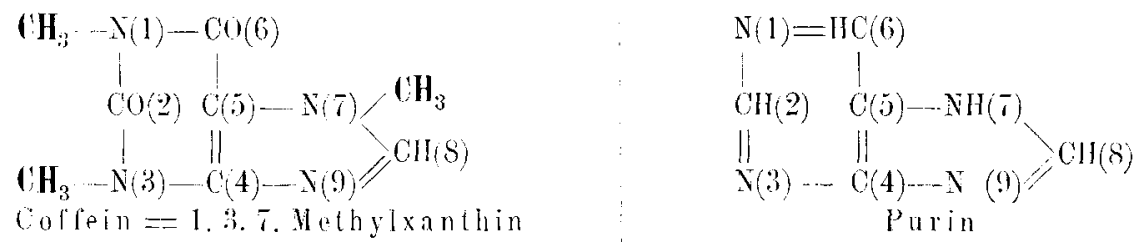

llir bezeishnen coffein num: Methyl 1. 6.

$$
\text { 2. э. } 8 \text {. Methyl }
$$

Methyl 3. 4. 9.

und die zunärehst geprüften Verbindungen:

Aethyl 1.6.

2. $5 . \%$

Methyl 3. 4. 9.

Aethylthoobromin
Methyl 1.6.

$$
\text { 2. 5. } 7 \text {. Methyl }
$$

Aethyl :3. 4. 9.

Acthyiparaxanthin
Mathyl 1.6

$$
\text { 2. 5. } 7 . \text { Aethyl }
$$

Methyl 3. 4. 9.

Aethyltheophyllin

Indem wir die ron den entspreehenden Dimethylxanthinen abgoledeten Namen hinzufügen, wird es verständlich, dass die linwartung einer Ibifferenz der medieinischen Wirkung nicht unberechtigt war. Wir laben gefunden: Alle drei Substanzen wirken ausgesprochen diuretiseh. Aethyltheophyllin steht jedoch quantitativ hinter lethyltheobromin zurïk beziiglich der quantitativen Verhälnisse muss der Uebersiehtlichkeit wegen auf die experimentellon Ingaben rerwiesen werden.)

Die Doppelsalze des Aethyltheobromins, das Natriumbenzoat und Satrimmalicylat wurden gleichfalls mehrfach geprift. Doch wollen wir auf diesc Binzolheiten vorläufow nicht cingehen.

Von diesen krystallisirten Canthinen, die sich ron Coffein nur dadurch unterscheiden, dass sie cine seince Methylgruppen durch die Aethylgruppe ersetat zeigen, haben wir nunmehr das in seiner ersten Stellung äthylirte, das Aethylhrobromin, verglichen mit densolben Vorbindungen, die in der ersten stollung andere Alkylreste tragen. Wir haben untersueht Propyl-, Butyl- und Amyltheobromin, und zwar von den beiden ersteren die normalen und Iso-Verbindungen, Amyltheobromin als IsoAmyltheobromin. Dis Bedentung der Seitenkette trit rielleicht boi folgender Aufzeidhung noch besser hervor.

$$
\begin{aligned}
& \begin{array}{ll|l}
\mathrm{CH}_{3} . \mathrm{CH}_{2} \cdot \mathrm{CH}_{2} \quad \text { 1.6. } & \mathrm{CH}_{3} \backslash \mathrm{CH}- & 1.6 . \\
\mathrm{CH}_{3} &
\end{array} \\
& \begin{array}{l|ll}
2.5 . & 7 . \\
\text { s. } & \mathrm{CH}_{3} & 2.5 .7 . \\
\hline & &
\end{array} \\
& \text { 3.4. } 9 . \\
& \text { Nornal-Propltheohromin : Iso-Propylheobromin }
\end{aligned}
$$




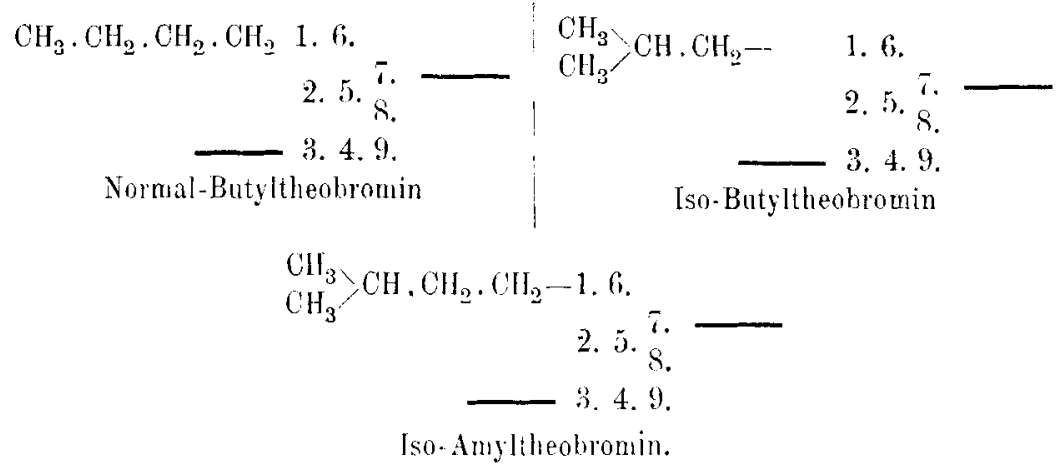

Alle diese alkylirten Theobromine wirken diuretiseth. Ls ist jodon sicher, dass die Lso-Amylverbindung beträehtlich zurücksteht. Wir sehliessen aus unseren Zahlen, dass die Länge der Scitenkette, aber crst von einer bestimmten Grenze an, die dinretische Nirkung an Intensität wesentlieh herabdrückt. Die lso-lerbindungen seheinen linter den normalen zurückzustehen.

Wir haben also gefunden für die ron uns untersuchten trialkylirten Xanthine:

Die Intensität der diuretischen W'irkung ist abhängig

1. bei den Vonö̈thyldimediylxanthinen (in der $1 ., 3 ., \quad 7$. stellungi ron der Isomerie.

2. bej den homologen 1. Mlkyl :3., 7. Dimethylxanthinen ron der Art des Alkylrestes.

Soweit unsere systematischen Intersuchungen, wie man sieht, nur ein goringer Theil cines grossen Gebietes.

IIr rerfügen dann noch über Einzeluntersuehungen, die in ilurem Ergebniss noeh interessantere Differenzen anfweisen für die theoretischen Tereleiche.

So haben wir noeh gefunden, dass ein Triathylxanthin $(1 ., 3 ., 7$. keine nennenswerthe diuretische Wirkung für die kranke Niere besitzt, ebensowenig 1., 3., 7. Methyl, 8. Nethylxanthin. Dagegen zeigt 3.. 7. Aethyl, 8. Methylxanthin eine beträchtliche diuretische Wirkung. Letzteres ist besonders ein Hinweis, wie vorsichtig man die Werthung der Zahl der einzclnen Acthyl- oder Methylgruppen rorzunehmen hat.

Ausser vom iethyltheobromin haben wir anch vom Propyltheobromin Doppelsalze dargestellt und intersucht. Im Mllgemeinen scheint es, dass die mit der Salzbildung Jinzugetretenen Eigensehaften für die Diurese mehr additiver, nicht principieller Satur sind. Iolekular berechnet zeigen die Salze ungelähr die Wirkung des Grundkörpers.

Ton Dimethylxanthinen war bereits das Diuretin eingehend miersucht. Vir haben nun auch ausser diesem 'Theobrominpräparat das Theophyllin (Theocin) geprüft. Es zejgte sich jedoch, dass diese beiden Dimethylxanthine in ihrer IIrkung aul die krantic Niere, wenigstens in den entsprechenden Dosen, nicht rergleichbar sind mit dem Coffein and den awälnten Homologen des Coffeins, hinter ibnen vielmohr erheblich zurückstehen. 
Ueb. d. Boziehungen zwischen chem. Constitution u. diuretischer Wirkung etc. 659

Vir brauchen wohl nicht hinzuzufügen, dass diese Art der Untersuchung ausschliesslich eine Ari quantitativer Prüfung der diuretischen IVirkmng eines Körpers auf die kranke Niere ist, aber krineswegs eine Vethode, die direet angiebt, ob ein Körper ein brauchbares Diureticom ist. \%weifollos werden sich bei den rersediedenen synthelisehen lingriften anch die übrigen physiologisehen Eigensehaften der Verbindungen, ror allem die (iifligkeit in mehr oder minder geselzmässiger lleise beeinllussen lassen. Wir suelen den Werth dieser neuen Prüfungsmethode hauptsä̈hlich darin, dass es möglich ist, aus einer so grossen Anzahl von Verbindungen ron rornherein nach wenigen Versuchen ganze Gruppen als ungeeignet auszuschalten und den lireis enger zu zichen. in dem sich die werthrollsten Individuen dieser pharmakologisels differenten Körperklasse wahrseheinlich befinden. Solbstrerständlich ist, dass die Reinheit der einzelnen Verbindungen genau controllirt wird. Fïr die isomeren Körper wird es wohl meist nöthg sein, die Einheitlichkeit dureh die biementamanalyse zo hestätigen, damit nicht gesetzmässige Unterselhede anligefunden werden, die in Mirkliehkeit auf eine unrollstandige Isolirmog ler lechindung zurickzufuhren sind.

Wir geben in kurzen Tabellen die wesentlichen Daten der wichtigston Verbindungen, welche wir verglichen haben. Die Versuchsanordnung glish der früher beschriebenen.

$$
\text { Acthyltheobromin } 1 \text { i. }
$$

\begin{tabular}{|c|c|c|c|c|}
\hline Datum & NaCl in Wasser & Uranlïsung injicirt & Medicament & Harnmenge \\
\hline 27. September & $1,0 / 25$ & $3 / 4$ Spritze 1 proc. & $0,0: 3$ & $30 \mathrm{ccm}$ \\
\hline 28. & $1,0 / 25$ & $n$ & 0,08 & 80 \\
\hline 29. & $1,0 / 25$ & $n$ & 0,03 & 18 \\
\hline ;30. & $1,0 / 25$ & . & 0,03 & kein Harn \\
\hline
\end{tabular}

1. Oktober: 'I'lier todt.

Section: Bauchhöhle $16 \mathrm{ccm}$, Brusthöhle $5 \mathrm{~cm}$

Controlthier: $\quad 100, \quad, \quad 40$,

Aethyltheobromin (grössere Dosis).

\begin{tabular}{|c|c|c|c|c|}
\hline 26. September & $1,0 / 25$ & $3 / 4$ Spritze I proc. & 0,04 & $116 \mathrm{ccm}$ \\
\hline 27. & $1,0 / 25$ & $n$ & 0,04 & $112 n$ \\
\hline 28. & $1,0 / 25$ & 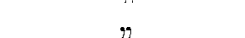 & 0,04 & 25 \\
\hline 29. & $1,0 / 25$ & $n$ & 0,04 & $-n$ \\
\hline
\end{tabular}

Thier tort. Section: Bauchhöhle $8 \mathrm{ccm}$, Brusthöhle $5 \mathrm{ccm}$.

$$
\text { Aethyltheobr. natr. benz. }
$$

\begin{tabular}{|c|c|c|c|c|}
\hline 19. Olituber & $1,0 / 25$ & $3 / 4$ Spritze 1 proe. & 0,05 & $140 \mathrm{ccm}$ \\
\hline 20. & $1,0 / 25$ & $\eta$ & 0,05 & 280 \\
\hline 21. & $1,0 / 25$ & . & 0,05 & 82 \\
\hline 22. & $1,0 / 2$ & . & 0,05 & $\begin{array}{r}n \\
-\quad n\end{array}$ \\
\hline 23 & $1,0 / 25$ &. & 0,05 & $30 n$ \\
\hline 24. & $1,0 / 25$ & " & 0,05 & 29 \\
\hline
\end{tabular}

$\left.\begin{array}{l}\text { Bauchhühle wenig, } 10 \mathrm{ccm} \text {, } \\ \text { Brusthühle ca } 25 \mathrm{~cm} \text {. Geringe Oedeme. }\end{array}\right\}$ Ilohe Irammengen.

Wirkung gut (kleine Dosis).

1) Die diuretisehe Wirkung der Aethyltheobromin wurde zuerst von Herm Dr. Martin kirüger festgestellt und untersucht. 
Aethyltheobr. natr. salicyl.

\begin{tabular}{|c|c|c|c|c|c|}
\hline Datum & NaCl in Wasser & Uranlösungr injicirt & Medicament & Harn & menge \\
\hline 19. Oktober & $1,0 / 25$ & 3/ Spritze 1 proc. & 0,05 & $\ldots$ & $\mathrm{ccm}$ \\
\hline 20. & $1,0 / 25$ & I & 0,05 & 270 & $x$ \\
\hline 21. & $1,0 / 25$ & $n$ & 0,05 & 62 &.. \\
\hline 22. & $1,0 / 25$ &. & 0,05 & $\ldots$ &. \\
\hline 23. & $1,0 / 25$ & י. & $0,0,5$ & 12 & .. \\
\hline 4. & $1,0 / 25$ & $\therefore$ & 0,05 & 6 & $\because$ \\
\hline
\end{tabular}

25. Oktober Thier toilt.

Bauchhöhle ca. $30 \mathrm{ccm}$

Brusthöhle ca. $30 \mathrm{ccm}$. Leichte Oedene.

Wirkung mässig (kileine Dosis).

Aethyltheobrom. natr. salioyl.

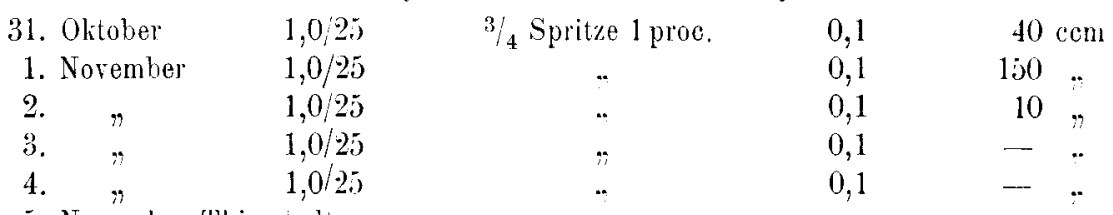

5. November 'lhier todt.

Im Bauch Spuren, Brust frei. Wirkung gut.

Normalpropyltheobromin.

\begin{tabular}{|c|c|c|c|c|c|}
\hline Datom & Kochsalz & Uranlösung injicirt & Nort & ropylth. & IIarnmenge \\
\hline 12. December & $1,0 / 25$ & $3 / 4$ Spritze 1 proc. & 0,1 & icament & \\
\hline 13. & $1,0 / 25$ & $\because$ & 0,1 & $\because$ & $74 \mathrm{cem}$ \\
\hline 4. & $1,0,25$ & 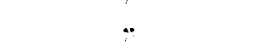 & 0,1 &. & 95, \\
\hline 5. & $1,0 / 25$ & $\eta$ & 0,1 & $\because$ & $2 n$ \\
\hline
\end{tabular}

Nachm. todt.

Section: Bauch- W. 1. Brusthöhle frei; vollkommen trocken. In der r. Brusthöhle $5 \mathrm{~cm}$ wenig blutgefübter Flüssigkeit. Keine Oedeme. Blase leer. Wirliung gut (grosse Dosis).

Normal-propyl-theobrom. natr. benz.

$\begin{array}{lcccc}\text { Datum } & \mathrm{YaCl} \text { in Wasser } & \text { Uranlösung injicirt } & \text { Medicament } & \text { Harnmenge } \\ \text { 4. Januar } & 1,0 / 25 & 3 / 4 \text { Spritze l proc. } & 0,1 & 18 \text { ccn } \\ 5 . & 1,0 / 25 & n & 0,1 & 8 \text { " } \\ 6 . & 1,0 / 25 & " & 0,1 & -\end{array}$

7. Januar: Jhier todt. Section: kein Ascites.

Iso-propyl-theobromin.

\begin{tabular}{|c|c|c|c|c|}
\hline 16. Decenber & $1,0 / 25$ & $3 / 4$ Spritze 1 proc. & 0,1 & $10 \mathrm{ccm}$ \\
\hline 17. & $1,0 / 25$ & $n$ & 0,1 & 60 \\
\hline 18. & $1,0 / 25$ & $\eta$ & 0,1 & 10 \\
\hline
\end{tabular}

19. December: Thier tot. Section: Bauch- und Brusthöhle trocken. Kein Ascites.

Därme trocken. Blase leer.

Normal-butyl-theobromin.

\begin{tabular}{|c|c|c|c|c|}
\hline 12. December & $1,0 / 25$ & $3 / 4$ Spritze 1 proc. & 0,1 & $24 \mathrm{ccm}$ \\
\hline 13. & $1,0 / 25$ & " & 0,1 & $70 \%$ \\
\hline 14. & $1,0 / 25$ & $"$ & 0,1 & 8 \\
\hline 15. & $1,0 / 25$ & 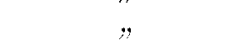 & 0,1 & kein Urin mehr \\
\hline
\end{tabular}

17. December: Thier todt. Section: Bauch- und Brusthöhle frei. Därne stark schwappend, mit Flüssigkeit gefüllt. Blase leer. 


\begin{tabular}{|c|c|c|c|c|}
\hline \multicolumn{5}{|c|}{ Iso-amyl-theobromin. } \\
\hline Datum & Kochsal\% & Uranlösung injicirt & Medicament & Harnmenge \\
\hline 16. December & $1,0 / 25$ & ${ }^{3}$ Spritze 1 proc. & 0,1 & $36 \mathrm{~cm}$ \\
\hline 17. & $1,0 / 25$ & , & 0,1 & 42, \\
\hline 18. & $1,0 / 25$ & $n$ & 0,1 & $\ldots$ \\
\hline 19. & $1,0 / 25$ & " & 0,1 & $5 \mathrm{ccm}$ \\
\hline 20. & 1,025 & $n$ & 0,1 & - \\
\hline
\end{tabular}

Thier tot. Kein Urin mehr. Section: Bauchhöhle $25 \mathrm{ccm}$, Brusthöhle $35 \mathrm{ccm}$.

Blase gefüllt.

Aethyl-theophyllin

$\begin{array}{lcccc}\text { 13. October } & 1,0 / 25 & 3 \text { Spritze } 1 \text { proc. } & 0,1 & 120 \mathrm{ccm} \\ 14 . " \# & 1,0 / 25 & " & 0,1 & 200 " " \\ 15 . \quad " & 1,025 & " & 0,1 & -\end{array}$

Abends: 'l'hier todt. Koin Urin. Section: Bauchhöhle: minimale Spuren Flüssigkeit. Brusthöhle nichts.

Aethyl-theophyllin.
4. Oetober
$1,0 / 25$
$3 / 4$ Spritze 1 proc.
$\begin{array}{rr}0,05 & 55 \mathrm{ccm} \\ 0,05 & 100 \quad 0\end{array}$
5. $, 1,0 / 25$
6. $\quad 1,0 / 25$
7. $\quad 1,0 / 25$
8. $" 1,0 / 25$
$(\therefore \mathrm{ccm}=0,03978 \mathrm{NaCl})$
0,05
$0,05 \quad$ kein Usin mehr
0,05 Thier frisst nicht

9. October: Thier tot. Section: Bauchhöhle $100 \mathrm{ccm}\left(\delta=0,90^{\circ}\right)$. Brusthöhle $42 \mathrm{~cm}\left(\delta=0,81^{\circ}\right)$. Kleinere Dosis $=0,05 \mathrm{~g}$, keine Wirkung.

Aethyl-paraxanthin.

\begin{tabular}{|c|c|c|c|c|}
\hline 24. November & $1,0 / 25$ & $3 / 4$ Spritze 1 proc. & 0,1 & $19 \mathrm{ccm}$ \\
\hline 25. & $1,0 / 25$ & , n & 0,1 & $5 n$ \\
\hline 26. & $1,0 / 25$ & $n$ & 0,1 & $30 "$ \\
\hline 27. & $1,0 / 25$ & $"$ & 0,1 & $20 " n$ \\
\hline 28. & $1,0 / 25$ & $n$ & 0,1 & $\ldots$ \\
\hline
\end{tabular}

29. November: Thier todt. Kein Lrin. Section: Kein Ascites, kein Hydrothorax. Theophyllin (Theocin).

\begin{tabular}{|c|c|c|c|c|}
\hline 21. Februar & $1,0 / 25$ & $3 / 4$ Spritze 1 proc. & 0,05 & $60 \mathrm{ccm}$ \\
\hline 22. & $1,0 / 25$ & $"$ & 0,05 & $\begin{array}{c}140 " \\
\text { Alb. }+ \text { Sacch. }\end{array}$ \\
\hline " & $1,0 / 25$ & $"$ & 0,05 & $36 \mathrm{ccm}$ \\
\hline$\because$ & $1,0 / 25$ & $n$ & 0,05 & Alb. +-; kein Harn \\
\hline , & $1,0 / 25$ & . & 0,05 & g \\
\hline$n$ & $1,0 / 25$ & , & 0,05 & 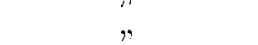 \\
\hline
\end{tabular}

27. Februar. Thier todt. Section: Keine Oedeme. Brusthöhle $35 \mathrm{ccm}$, Bauchhöhle $110 \mathrm{com}$ klare Hlïssigkeit. B]ase leer. - Zweiter Versuch mit 0,1 Theocin pro die. Thier am 3. Tage todt.

$$
\text { Angewandte Verbindungen }{ }^{1} \text { ). }
$$

Aethyl-theobromin Smp. $166-167^{\circ}\left\{\begin{array}{c}\text { Löslich in Wasser ron } 15^{\circ} \text { inca.35Theilen } \\ " \text { "siedendem Wasser in } 3 " n\end{array}\right.$

1) Die genaue Beschreibung dieser z. Th. neuen chemischen Verbindungen wird an anderer Stelle erfolgen. 
N-P'ropyl-theobromin Iso-Propyl-theobromin N.Butyl-theobronin Iso-Butyl-theobromin Iso-Amyl-theobromin Aethyl-theophyllin

$\&$ Methyl 3,7 Dimethylxantlin

8 Methyl 13, Triäthylxanthin Aethyl-theobromin $\mathrm{C}_{9} \mathrm{I}_{12} \mathrm{~N}_{4} \mathrm{O}_{2}$

$0,1731 \mathrm{~g}$ Substanz gaben $0,3283 \mathrm{~g} \mathrm{CO}_{2} ; 0,0404 \mathrm{~g} \mathrm{H}_{2} \mathrm{O}$.

Authyl-theophyllin $\mathrm{C}_{9} " \mathrm{H}_{12} \mathrm{~N}_{4} \mathrm{O}_{4}$

Sup. $137,5-138^{\circ}$ L.̈̈sl. in 40 Th. W.; in 3 Th. h. W.

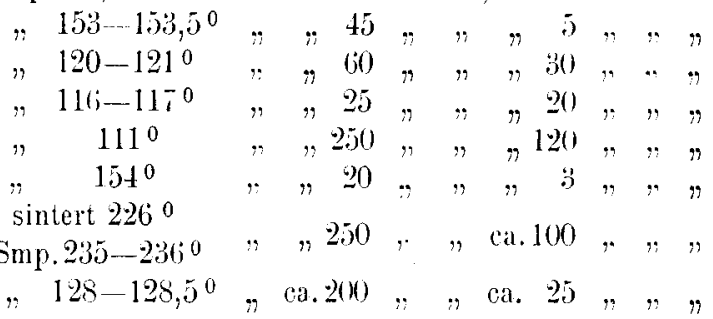

C. ber. 51,91 pCt., gef. $51,72 \mathrm{pCt}$.

H. $" 5,7 \% "$ " 5,80 "

$0,1781 \mathrm{~g}$ Substanz gaben $0,3384 \mathrm{~g} \mathrm{CO}_{2} ; 0,0905$ g $\mathrm{H}_{2} \mathrm{O}$.

C. ber. $51,91 \mathrm{pCt}$, gef. $51,82 \mathrm{pCt}$.

Triäthylxanthin $\mathrm{C}_{11} \mathrm{H}_{16} \mathrm{~N}_{4} \mathrm{O}_{2}$

H. $\quad 5,77$ " $\quad 5,64$ "

$0,1800 \mathrm{~g}$ Substanz gaben $0,3660 \mathrm{~g} \mathrm{CO}_{2,} ; 0,10 \mathrm{~s}$ g $\mathrm{H}_{2} \mathrm{O}$.

C. ber. 55,93 pCt., gef. $55,45 \mathrm{pCt}$.

H. $" 6,78$ n $\quad 6,08$ " 